\title{
Comparison of the Utility of Recombinant B8/2 Subunit of the Antigen B, Native Antigen, and a Commercial ELISA Kit in the Diagnosis of Human Cystic Echinococcosis
}

\author{
Amir Savardashtaki ${ }^{1}$, Zohreh Mostafavi-Pour ${ }^{2}$, Farzaneh \\ Arianfar $^{2}$ and Bahador Sarkari ${ }^{3^{*}}$ \\ ${ }^{1}$ Department of Medical Biotechnology, School of Advanced Medical Sciences and Technologies, \\ Shiraz University of Medical Sciences, Shiraz, Iran; ${ }^{2}$ Recombinant Proteins Laboratory, \\ Department of Biochemistry, School of Medicine, Shiraz University of Medical Sciences, Shiraz, Iran; \\ ${ }^{3}$ Department of Parasitology and Mycology, School of Medicine, Shiraz University of Medical Sciences, Shiraz, Iran
}

Received 4 April 2018; revised 2 June 2018; accepted 24 July 2018

\begin{abstract}
Background: Cystic echinococcosis (CE) is a helminthic disease caused by the larval form of Echinococcus granulosus. In the present study, the B8/2 subunit of antigen $\mathrm{B}(\mathrm{AgB})$ of $E$. granulosus was expressed in $E$. coli host and then applied in a diagnostic ELISA set up. Methods: The DNA sequence of AgB8/2 subunit from $E$. granulosus was extracted from the GenBank and codon-optimized according to $E$. coli codon usage. The target sequence was cloned in an expression vector (pGEX-4T-1). The produced antigen was used in an ELISA system, and its performance for the diagnosis of human hydatid cyst was evaluated, using sera from CE and non-CE patients, along with the sera from healthy subjects. Moreover, the diagnostic value of the recombinant protein was compared with native $\mathrm{AgB}$, as well as with a commercial kit. Results: Antibodies to hydatid cyst were detected in 27 out of 30 patients corresponding to a sensitivity of $90 \%$ ( $95 \% \mathrm{Cl}$ : 73-98\%). Cross-reaction with sera of non-CE subjects was seen in two cases resulted in a specificity of $93.5 \%$ (95\% Cl: $82-98 \%$ ) for the test. A sensitivity of $87 \%$ and specificity of $90 \%$ were found for the native form of the antigen, while the ELISA commercial kit had a sensitivity of $97 \%$ and specificity of $95 \%$. Conclusion: Our data show that $\mathrm{rEgAgB} 8 / 2$ is an appropriate source of antigen for the serological diagnosis of human hydatid cyst. Co-expression of the $\mathrm{rEgAgB} / 2$ along with other subunits of $\mathrm{AgB}$ may enhance the performances of these antigens for the serodiagnosis of human CE. DOI: 10.29252/ibj.23.4.246
\end{abstract}

Keywords: Diagnosis, Echinococcus granulosus, Hydatid cyst

\section{INTRODUCTION}

$\mathrm{C}$ ystic echinococcosis (CE), or hydatid cyst, is a chronic parasitic disease that is caused by the larval form or metacestode of Echinococcus granulosus $^{[1]}$. The cyst is mainly localized in human liver and lung, and its clinical presentation may range from an asymptomatic infection to a severe and fatal disease $^{[1,2]}$. CE is considered as an important economic and public health concern in several developing countries including Iran ${ }^{[1,3]}$. This disease affects about 1.2 million people worldwide, and the global burden of the disease is estimated to be 188,000 new cases per year ${ }^{[1,3]}$. In Iran, the annual number of CE surgeries has been reported to be 1295 cases, accounting for a mean annual surgical incidence of 1.6 in 100,000 inhabitants ${ }^{[4,5]}$. Seroepidemiological surveys have indicated asymptomatic CE in $7.3 \%$ of the individuals 
in the rural areas of Iran's southeastern province of Kerman and $5.6 \%$ in the volunteer blood donors from Fars Province in southern $\operatorname{Iran}^{[6,7]}$. CE diagnosis is often confirmed by a combination of imaging techniques along with serological approaches. So far, several serological methods, based on antigen or antibody detection, have been utilized for the diagnosis and follow-up of $\mathrm{CE}$ with satisfactory results, but far from being optimal due to their suboptimal sensitivities or specificities ${ }^{[1,8-12]}$.

The development of DNA recombinant technology has facilitated the generation of recombinant peptides suitable for highly sensitive approaches in the diagnosis of different infectious diseases including $\mathrm{CE}^{[13-16]}$. Several antigens of E. granulosus have been evaluated in different serodiagnosis assays of human hydatid cyst ${ }^{[8,13]}$. Among them, antigen 5 (Ag5) and antigen $\mathrm{B}(\mathrm{AgB})$ are frequently used antigens. $\mathrm{AgB}$, known as the main protein responsible for metabolic adaptation and survival of the parasite, is the prominent antigen of hydatid cyst fluid. The antigen is a lipoprotein of 120-160 kDa, consisted of a group of about $8-\mathrm{kDa}$ subunits. Under reducing conditions in SDS-PAGE, it dissociates into 8, 16, and $24 \mathrm{kDa}$ subunits ${ }^{[17,18]}$. The $8-\mathrm{kDa}$ subunit, the smallest one, has presented the best performance in the diagnosis of $\mathrm{CE}$ and has been extensively used in synthetic, recombinant, or native forms in different serological assays $^{[10,12,14,19-21]}$.

Molecular studies have demonstrated that $\mathrm{AgB}$ is encoded by a multigene family that is variably expressed, with at least five major gene clusters named EgAgB1-5 ${ }^{[18]}$. The putative protein isoforms encoded by the five $\mathrm{EgAgB}$ genes differ $44-81 \%$ in amino acid sequence $^{[18]}$. A comparison between the $\mathrm{AgB} 8 / 1$ and AgB8/2 nucleotide sequences has showed $53.5 \%$ identity among exons and $50 \%$ identity between introns of these two subunits ${ }^{[22]}$. Accordingly, the immunodiagnostic performances of different subunits of $\mathrm{AgB}(\mathrm{EgAgB} 8 / 1-5)$ seem to be different. Formerly, it has been demonstrated that the recombinant EgAgB8/2 (rEgAgB8/2) has a higher diagnostic value in comparison with the $\mathrm{rEgAgB} 8 / 1$ or native $\mathrm{AgB}$ for the serodiagnosis of human $\mathrm{CE}^{[22]}$.

In our previous study, we successfully cloned and expressed the $\mathrm{EgAgB} 8 / 1$ subunit of $\mathrm{AgB}$ and evaluated its diagnostic efficacy in CE. A sensitivity and specificity of $93 \%$ and $92 \%$ was found for the EgAgB8/1 subunit ${ }^{[14]}$. In the current study, we focused on other subunits of the antigen where the $\mathrm{EgAgB} 8 / 2$ was expressed in $E$. coli host, and its performance for serological diagnosis of human $\mathrm{CE}$ was evaluated using an ELISA system.

\section{MATERIALS AND METHODS}

\section{Construction, optimization, and cloning of $\mathrm{AgB8} / 2$}

Construction, optimization, and cloning of rEgAgB8/2 were performed as previously described with some modifications ${ }^{[14]}$. Briefly, the DNA sequence of $\mathrm{AgB} 8 / 2$ was extracted from NCBI GenBank database with the accession number of DQ835667.1. The target sequence was optimized based on $E$. coli codon usage and the optimized gene fragment was synthesized (Biomatik, Ontario, Canada) and cloned in pBluescript II SK (+) vector. The fragment was subsequently excised by EcoRI/XhoI digestion and subcloned in pGEX-4T-1 (GE Healthcare, Illinois, US) expression vector and transformed into $E$. coli DH5 $\alpha$. The recombinant plasmid was extracted using QIAprep Spin Miniprep Kit (Qiagen, Hilden, Germany). The extracted plasmid was transformed into BL21 (DE3)pLysS cells for expression under the $\mathrm{T} 7$ promoter. The expression of $\mathrm{AgB} 8 / 2$ was induced by $0.5 \mathrm{mM}$ IPTG (Invitrogen, USA) at $37^{\circ} \mathrm{C}$ for four hours.

\section{Protein purification}

The cells were harvested by centrifugation $(1800 \times \mathrm{g}$ at $4{ }^{\circ} \mathrm{C}$ for $10 \mathrm{~min}$ ), and the resultant pellet was resuspended in a lysis buffer (Triton-X100, PBS [pH 8], and $0.5 \mathrm{M}$ EDTA) and sonicated $(5 \times 30 \mathrm{~s})$ on ice. The resultant cell lysate was centrifuged at $15,000 \times \mathrm{g}$ at 4 ${ }^{\circ} \mathrm{C}$ for $30 \mathrm{~min}$ and incubated at $-20^{\circ} \mathrm{C}$ overnight. After that, the clear supernatant was collected, and the recombinant protein was purified by affinity chromatography, based on its glutathione S-transferase (GST)-tag, using immunoaffinity column (SigmaAldrich, Germany). The bound protein was eluted with elution buffer (reduced glutathione, $50 \mathrm{mM}$ Tris, $\mathrm{pH}$ $8)$. Finally, desalination of purified protein was done using a dialysis membrane in PBS $(\mathrm{pH} 7.5)^{[14]}$.

\section{SDS-PAGE and Western blotting}

SDS-PAGE of the recombinant and the native protein (native $\mathrm{AgB}$ purified from hydatid cyst fluids as described previously) ${ }^{[21]}$ were carried out on $18 \%$ (w/v) polyacrylamide gel containing $0.1 \%$ SDS as documented before ${ }^{[14]}$.

\section{Patients and control sera}

Hydatid cyst patients' sera were obtained from 30 pathologically-confirmed CE patients. In addition, 32 samples of non-CE patients, including subjects affected by giardiasis $(\mathrm{n}=3)$, hymenolepiasis $(\mathrm{n}=3)$, toxocariasis $(n=3)$, toxoplasmosis $(n=3)$, fascioliasis $(\mathrm{n}=3)$, malaria $(\mathrm{n}=2)$, cryptosporidiosis $(\mathrm{n}=2)$, trichostrongyliasis $(n=1)$, patients with autoimmune 


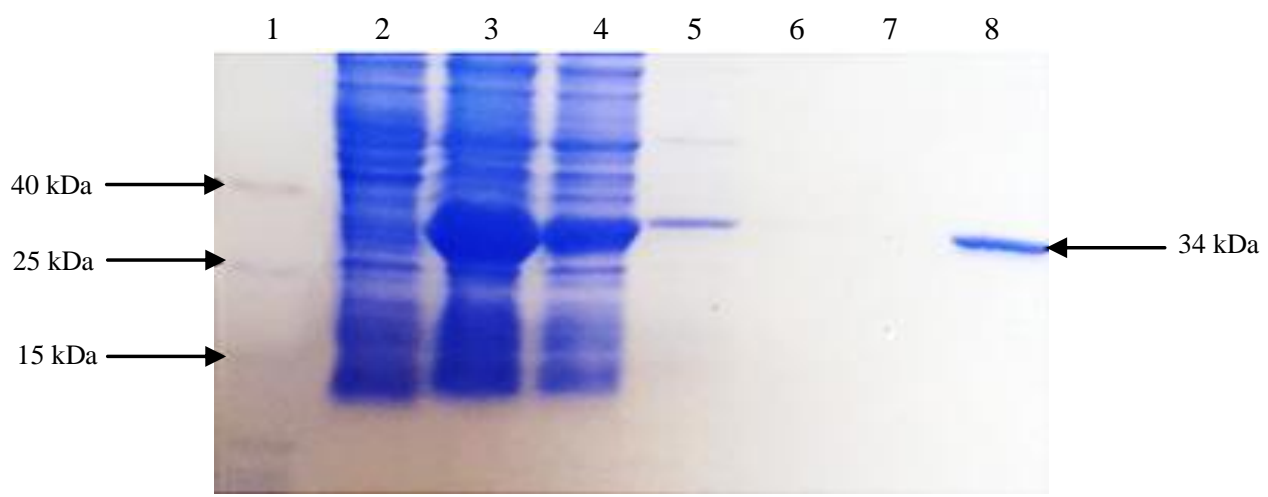

Fig. 1. SDS-PAGE analysis of the $\mathrm{rEgAgB} 8 / 2$ protein expression and purification. Lane 1, protein weight marker; lanes 2 and 3 , uninduced and induced cultures of $E$. coli by IPTG; lane 4, prewash; lanes 5, 6, and 7, consecutive washes; lane 8, final elution (GST$\mathrm{AgB} 8 / 2)$.

diseases $(n=9$; including three with vitiligo, three with Behçet's disease, and three with systemic lupus erythematous) as well as patients with FUO (fever of unknown origin; $\mathrm{n}=3$ ) were collected. Moreover, 30 sera were collected from the healthy subjects who had no history of $\mathrm{CE}$ and were all seronegative when tested for anti-hydatid cyst antibodies, using AgB-ELISA ${ }^{[10]}$. The study was approved by the Ethical Committee of Shiraz University of Medical Sciences (Ethical code number: IR.SUMS.REC. 1393.S7376), and an inform consent was obtained from those who voluntarily donated the samples or from their patients.

\section{ELISA using native and recombinant antigen}

ELISA with native $\mathrm{AgB}$ and $\mathrm{rEgAgB} 8 / 2$ was performed as previously described ${ }^{[14]}$. Briefly, 96-well microplates were sensitized with $5 \mu \mathrm{g} / \mathrm{mL}$ of either rEgAgB8/2 or native $\mathrm{AgB}$. Unbound sites were blocked with non-fat skimmed milk. Sera samples of $\mathrm{CE}$ patients along with positive and negative controls were added to the microplates. Conjugated goat antihuman $\operatorname{IgG}$ was used to detect the antigen-antibody complex and a substrate (o-phenylenediamine; 0.4 $\mathrm{mg} / \mathrm{mL}$ ) was used to visualize the reaction. The cut-off point was considered as $2 \mathrm{SD}$ above the mean of control samples.

\section{ELISA with the commercial kit}

All the sera samples were analyzed by a commercial ELISA kit (Euroimmune, Germany), available for the diagnosis of $\mathrm{CE}$, based on the manufacturer's guidelines.

\section{Statistical analysis}

The diagnostic accuracy of the native $\mathrm{AgB}$, $\mathrm{rEgAgB} / 2$, and the commercial kit was verified by the area under the receiver operating characteristic curve, using SPSS software (ver. 20). Cut-off values were established for the test based on Youden's Index. Cohen's kappa statistic was used to find out the extent of agreement between ELISA systems, using different antigens, and also the commercial kit.

\section{RESULTS}

\section{Production and purification of $\mathrm{rEgAgB8} / 2$}

After cloning a part of the $\mathrm{AgB}$ along with the signal sequence $(\mathrm{rEgAgB} / 2)$ in a pGEX-4T-1 vector, the correct cloning procedure was confirmed through the sequencing of the expression unit. The expression of the $\mathrm{rAgB} 8 / 2$ with the estimated molecular weight of 34 $\mathrm{kDa}$ was confirmed by SDS-PAGE analysis (Fig. 1). Reactivity of the recombinant protein with sera of $\mathrm{CE}$ patients was further confirmed with Western blotting (Fig. 2). A GST agarose resin was used for the purification of the developed recombinant protein.

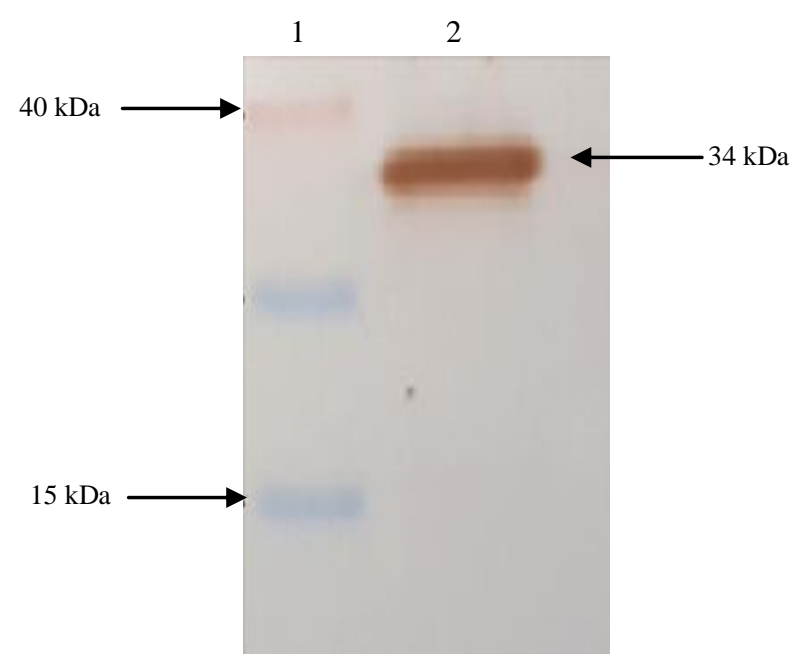

Fig. 2. Western blotting analysis of $\mathrm{rEgAgB} 8 / 2$ expression in E. coli. Lane 1, molecular weight marker; Lane 2, $\mathrm{rEGAgB} 8 / 2$ fused with GST tag. 
Table 1. The performance of $\mathrm{rEgAgB} 8 / 2$, native antigen $\mathrm{B}$, and commercial kit for the diagnosis of CE

\begin{tabular}{lcccc}
\hline Type of serum & Number & $\begin{array}{c}\text { Positive cases in } \\
\text { commercial kit } \\
\text { No. }(\%)\end{array}$ & $\begin{array}{c}\text { Positive cases in } \\
\text { native-AgB ELISA } \\
\text { No. }(\%)\end{array}$ & $\begin{array}{c}\text { Positive cases in } \\
\text { rEgAgB8/2 ELISA } \\
\text { No. (\%) }\end{array}$ \\
\hline CE patients & 30 & $29(97)$ & $26(87)$ & $27(90)$ \\
Vitiligo & 3 & 0 & 0 & 0 \\
Behçet's disease & 3 & 0 & 0 & 0 \\
Lupus & 3 & 0 & 0 & 0 \\
Giardiasis & 3 & 0 & $1(33)$ & 0 \\
Hymenolepiasis & 3 & 0 & 0 & 0 \\
Toxocariasis & 3 & $1(33)$ & 0 & 0 \\
Toxoplasmosis & 3 & 0 & $1(33)$ & $1(33)$ \\
Fascioliasis & 3 & 0 & $1(33)$ & $1(33)$ \\
FUO & 3 & 0 & $1(33)$ & 0 \\
Malaria & 2 & $1(50)$ & 0 & 0 \\
Cryptosporidiosis & 2 & 0 & 0 & 0 \\
Trichostrongyliasis & 1 & 0 & 0 & 0 \\
Healthy subjects & 30 & $1(3.3)$ & $2(6.7)$ & $2(6.7)$ \\
\hline
\end{tabular}

\section{Diagnosis of human CE using rEgAgB8/2}

GST-containing $\mathrm{rEgAgB} / 2$ was used in the ELISA system to check its performance for the detection of the anti-hydatid cyst antibodies. Using an ELISA checkerboard, the optimum concentrations of the $\mathrm{rEgAgB} 8 / 2$, horseradish peroxidase-conjugated IgG, and the serum were determined as $5 \mu \mathrm{g} / \mathrm{mL}, 1: 100$, and 1:4000 dilutions, respectively. Using the developed ELISA system, anti-hydatid cyst antibodies were detected in the sera of 27 out of $30 \mathrm{CE}$ patients corresponding to a sensitivity of $90 \%$ (95\% CI: 73 98\%). The test had a specificity of $93.5 \%$ (95\% CI: 82$98 \%$ ) since cross-reaction with non-CE cases was seen in 2 out of 30 samples. None of the healthy subjects were positive by the developed ELISA system. The performance of the ELISA, using the $\mathrm{rEgAgB} 8 / 2$, the ELISA commercial kit, and native $\mathrm{AgB}$ for the diagnosis of human hydatid cyst are shown in Table 1. Native AgB was able to detect anti-hydatid cyst antibodies in the sera of 26 out of $30(86.7 \%)$ of $\mathrm{CE}$ cases. Six cases of controls were also positive in AgBELISA. Accordingly, a sensitivity of $87 \%$ and specificity of $90 \%$ were calculated for the native AgB. A sensitivity of $97 \%$ and specificity of $95 \%$ were also found for the ELISA commercial kit. The performances of the $\mathrm{rEgAgB} 8 / 2$, native antigen, and the commercial kit for the diagnosis of $\mathrm{CE}$ are shown in Table 2. There was a good agreement among the three assays. The kappa coefficient $(k)$ for $\mathrm{rEgAgB} 8 / 2$ and native $\mathrm{AgB}$ was 0.786 , for native $\mathrm{AgB}$ and the commercial kit was 0.76 , and for $\operatorname{rEgAgB} 8 / 2$ and the commercial kit was 0.83 .

\section{DISCUSSION}

The primary asymptomatic CE may last for years and the late diagnosis may be associated with inadequate care, resulting in the disease spread and poor clinical outcomes. In contrast, timely diagnosis of the CE offers better opportunities for timely intervention and control of the disease. Diagnosis of CE is based on imaging modalities along with serological approaches ${ }^{[8]}$. Serological assays along with the imaging approaches would confirm the diagnosis of CE. Low sensitivity and also low specificity render the optimal performance of such approaches for proper diagnosis of the disease.

To overcome the problem of low sensitivity and cross-reactivity, utilizing the recombinant antigens in the diagnosis of hydatid cyst has gained much attention ${ }^{[23,24]}$. In the previous study, we produced a recombinant antigen, $\mathrm{AgB} 8 / 1$, as a primary target for the development of an immunoassay for the diagnosis CE with satisfactory results ${ }^{[14]}$. In the current study, production of $\mathrm{EgAgB} 8 / 2$, as a specific and highly abundant antigen of E. granulosus was considered. The five subunits of $\mathrm{AgB}$ have relatively same molecular weights, but the amino acid sequences of these subunits differ as much as $44-81 \%$. Thus, their performances in the immunodiagnosis of hydatid cyst seem to be different. Using several approaches, a high level of the heterologous expression of the $\mathrm{EgAgB} 8 / 2$ fragment was achieved. We effectively take advantage of codon optimization to get a high-level expression of $\mathrm{EgAgB} 8 / 2$ as a eukaryotic antigen in $E$. coli as a prokaryotic cell factory. 
Table 2. Relative performances of the $\mathrm{rEgAgB} 8 / 2$, the native antigen, and the ELISA commercial kit for the diagnosis of CE

\begin{tabular}{lcccc}
\hline Type of antigens & $\begin{array}{c}\text { Sensitivity (\%) } \\
(\mathbf{9 5 \%} \text { CI) }\end{array}$ & $\begin{array}{c}\text { Specificity (\%) } \\
(\mathbf{9 5 \%} \text { CI) }\end{array}$ & $\begin{array}{c}\text { PPV (\%) } \\
(\mathbf{9 5 \%} \text { CI) }\end{array}$ & $\begin{array}{c}\text { NPV (\%) } \\
(\mathbf{9 5 \%} \text { CI) }\end{array}$ \\
\hline rEgAgB8/2 & $90(73-98)$ & $93.5(82-98)$ & $88(67-95)$ & $95(86-99)$ \\
Native AgB & $87(68-95)$ & $90(79-96)$ & $81(63-92)$ & $93(83-98)$ \\
Commercial kit & $97(81-100)$ & $95(85-99)$ & $90(74-97)$ & $98(90-100)$ \\
\hline
\end{tabular}

PPV, positive predictive value; NPV, negative predictive value

In our pilot study, at first, $\mathrm{AgB} 8 / 2$ was used without codon optimization but proper expression level of the desired protein was not achieved. As it has been shown in previous studies ${ }^{[25-27]}$ the codon optimization increases the heterologous expression of the recombinant proteins in E. Coli. Hence, we decided to optimize AgB8/2 DNA for bacterial expression, using Genscript's OptimumGene ${ }^{\mathrm{TM}}$ design platform, which employs a unique algorithm and proprietary codon usage table. Besides, codon adaptation index was one of the parameters analyzed in our study. When this index is more than 0.8 , a high expression level of protein can be expected. For our protein, this index was 0.4 before codon optimization and increased to 0.9 after that. The other significant factor is codon preference. The most frequent codons worth 100, and when the frequency of these codons increases in one sequence, the chance of expression increases as well. This value was $44 \%$ for $\mathrm{AgB} 8 / 2$ before codon optimization and after that was improved up to $75 \%$. Based on above optimization, a final concentration of 4 $\mathrm{mg} / \mathrm{mL}$ of the purified $\mathrm{EgAgB} 8 / 2$ protein was obtained, which is higher than the yield of $\mathrm{AgB} 8 / 1$ in our previous study ${ }^{[14]}$.

The antigen was expressed as a GST-tagged fusion protein to enable GST affinity purification, enhance protein expression and increase the solubility of the produced protein. It has been shown that the nterminus fusion of the target recombinant protein with one of the host proteins will increase the yield of expression as well as its solubility and reduces the development of inclusion bodies ${ }^{[28-30]}$. Considering this point, in the present study, the protocol for native purification was followed to keep the native construction of the protein and also to prevent denaturing of the protein and to keep the intact conformational epitopes. Enzymatic cleavage, by thrombin, can simply be used to remove the tag. No differences in the immunoreactivity of the antigens were seen when the $\mathrm{rEgAgB} 8 / 2$, with or without tag, was evaluated for the detection of anti-hydatid cyst antibodies. Therefore, we assumed that fusion tag did not interfere in the antigen-antibody reaction in our ELISA system. In order to be in a safe side and to avoid the antigen degradation or possible alteration in the conformational epitopes of the antigen by enzymatic reactions, which are necessary for the cleavage of the tag, we left the antigen coupled with the tag. Furthermore, immunoreactivity analysis revealed that GST tag usually does not affect the performance of the tests since they do not cross-react with antibodies present in the human serum.

The sensitivity and specificity of the produced $\mathrm{EgAgB} 8 / 2$ antigen in this study were $90 \%$ and $93.5 \%$, respectively, which were higher than those of the native counterpart. It appears that pairing this antigen with $\mathrm{EgAgB} 8 / 1$ antigen may improve the performance of the test for the diagnosis of human hydatid cyst. Mohammadzadeh et al. $^{[12]}$ have achieved similar results with recombinant antigen from Japanese isolates of E. granulosus. In a study in China, Jiang et $a l .{ }^{[30]}$ have indicated that the sensitivity of the $\mathrm{EgAgB} 8 / 1$ antigen is greater than that of $\mathrm{EgAgB} 8 / 2$ in the diagnosis of human $\mathrm{CE}$. Comparison of the performances of $\mathrm{EgAgB} 8 / 1$ produced in our previous study with $\operatorname{EgAgB} 8 / 2$ subunits revealed that the sensitivity of $\mathrm{AgB} 8 / 1$ antigen is greater than $\mathrm{AgB} 8 / 2$. This result is consistent with Jiang et al. $\mathrm{s}^{[30]}$ study in which they reported a higher sensitivity for $\mathrm{AgB} 8 / 1$ in comparison with $\mathrm{AgB} / 2^{[30]}$.

Pairwise comparison of the immunoreactivity results of the sera with $\mathrm{AgB} 8 / 1$ and $\mathrm{AgB} 8 / 2$ demonstrated that some samples are reactive with one of these antigens but not with the other one. This situation may reflect the different status of the cyst in the host and the dominance of the expression of one of AgB subunits. Recently, immunoproteomics studies has demonstrated a difference in the expression of immunogenic antigens of hydatid cysts in the CE1 and CE2 stages where it was found that in the CE1 stage, the $\mathrm{AgB} 8 / 1$ expression level was higher, while in the CE2 stage, the expression level of $\mathrm{AgB} 8 / 2$ was greater ${ }^{[31,32]}$. This claim may describe why some sera samples are reactive with $\mathrm{AgB} 8 / 1$ but not with $\mathrm{AgB} 8 / 2$ antigen ${ }^{[22]}$.

Findings of the current study reveal that gene codon optimization and fusion with GST tag sequence facilitate both the heterologous expression of the $\mathrm{rAgB} 8 / 2$ antigen in $E$. coli and its purification. The purified recombinant protein is a good antigenic source for the serological diagnosis of CE. Co-expression of 
the $\mathrm{EgAgB} / 2$ along with $\mathrm{EgAgB} / 1$ and application of these two proteins in an ELISA system may improve the performances of these recombinant antigens in the diagnosis of CE. Further studies are needed to clarify the diagnostic value of other subunits of $\mathrm{AgB}$, particularly $\mathrm{EgAgB} / 4$, utilizing a native strain of $E$. granulosus.

\section{ACKNOWLEDGMENTS}

This study was financially supported by the Office of Vice-Chancellor for Research of Shiraz University of Medical Sciences (Grant No. 93-7376), Shiraz, Iran. The results described in this paper were a part of Ph.D. student thesis of Amir Savardashtaki.

CONFLICT OF INTEREST. None declared.

\section{REFERENCES}

1. Kern P, Menezes da Silva A, Akhan O, Müllhaupt B, Vizcaychipi KA, Budke C, Vuitton DA. The Echinococcoses: Diagnosis, clinical management and burden of disease. Advances in parasitology 2017; 96: 259-369.

2. Sarkari B, Fatemie Sfedan A, Moshfe A, Abdolahi Khabisi S, Savardashtaki A, Hosseini F, Shahbazi A. Clinical and molecular evaluation of a case of giant primary splenic hydatid cyst: A case report. Iranian journal of parasitology 2016; 11(4): 585-590.

3. Deplazes P, Rinaldi L, Alvarez Rojas CA, Torgerson PR, Harandi MF, Romig T, Antolova D, Schurer JM, Lahmar S, Cringoli G, Magambo J, Thompson RC, Jenkins EJ. Global distribution of alveolar and cystic echinococcosis. Advances in parasitology 2017; 95: 315-493.

4. Fasihi Harandi M, Budke CM, Rostami S. The monetary burden of cystic echinococcosis in Iran. PLoS neglected tropical diseases 2012; 6(11): e1915.

5. Sarkari B, Sadjjadi SM, Beheshtian MM, Aghaee M, Sedaghat F. Human cystic echinococcosis in Yasuj District in Southwest of Iran: an epidemiological study of seroprevalence and surgical cases over a ten-year period. Zoonoses and public health 2010; 57(2): 146150

6. Harandi MF, Moazezi SS, Saba M, Grimm F, Kamyabi H, Sheikhzadeh F, Sharifi I, Deplazes P. Sonographical and serological survey of human cystic echinococcosis and analysis of risk factors associated with seroconversion in rural communities of Kerman, Iran. Zoonoses and public health 2011; 58(8): 582-588.

7. Sarkari B, Hosseini F, Khabisi SA, Sedaghat F. Seroprevalence of cystic echinococcosis in blood donors in Fars province, southern Iran. Parasite epidemiology and control $2016 ; 2(1): 8-12$.
8. Sarkari B, Rezaei Z. Immunodiagnosis of human hydatid disease: where do we stand? World journal of methodology 2015; 5(4): 185-195.

9. Siles-Lucas M, Casulli A, Conraths FJ, Müller N. Laboratory diagnosis of Echinococcus spp. in human patients and infected animals. Advances in parasitology 2017; 96: 159-257.

10. Sadjjadi SM, Abidi H, Sarkari B, Izadpanah A, Kazemian S. Evaluation of enzyme linked immunosorbent assay, utilizing native antigen B for serodiagnosis of human hydatidosis. Iranian journal of immunology 2007; 4(3): 167-172.

11. Sadjjadi SM, Sedaghat F, Hosseini SV, Sarkari B. Serum antigen and antibody detection in echinococcosis: application in serodiagnosis of human hydatidosis. Korean journal of parasitology 2009; 47(2): 153-157.

12. Mohammadzadeh T, Sako Y, Sadjjadi SM, Sarkari B, Ito A. Comparison of the usefulness of hydatid cyst fluid, native antigen $\mathrm{B}$ and recombinant antigen B8/1 for serological diagnosis of cystic echinococcosis. Transactions of the royal society of tropical medicine and hygiene 2012; 106(6): 371-375.

13. Carmena D, Benito A, Eraso E. Antigens for the immunodiagnosis of Echinococcus granulosus infection: An update. Acta tropica 2006; 98(1): 74-86.

14. Savardashtaki A, Sarkari B, Arianfar F, Mostafavi-Pour Z. Immunodiagnostic value of Echinococcus granulosus recombinant b8/1 subunit of antigen B. Iranian journal of immunology 2017; 14(2): 111-122.

15. Hemmati M, Seghatoleslam A, Rasti M, Ebadat S, Mosavari N, Habibagahi M, Taheri M, Sardarian AR, Mostafavi-Pour Z. Expression and purification of recombinant Mycobacterium tuberculosis (TB) antigens, ESAT-6, CFP-10 and ESAT- 6/CFP-10 and their diagnosis potential for detection of TB patients. Iraninan red crescent medical journal 2011; 13(8): 556563.

16. Movahedi B, Mokarram P, Hemmati M, Mosavari N, Zare R, Ardekani LS, Mostafavi-Pour Z. IFN- $\gamma$ and IL-2 responses to recombinant AlaDH against ESAT-6/CFP10 fusion antigens in the diagnosis of latent versus active tuberculosis infection. Iranian journal of medical sciences 2017; 42(3): 275-283.

17. Folle AM, Kitano ES, Lima A, Gil M, Cucher M, Mourglia-Ettlin G, Iwai LK, Rosenzvit M, Batthyany C, Ferreira AM. Characterisation of antigen B Protein species present in the hydatid cyst fluid of Echinococcus canadensis G7 Genotype. PLoS neglected tropical diseases 2017; 11(1): e0005250.

18. Monteiro KM, Cardoso MB, Follmer C, da Silveira NP, Vargas DM, Kitajima EW, Zaha A, Ferreira HB. Echinococcus granulosus antigen B structure: subunit composition and oligomeric states. PLoS neglected tropical diseases 2012; 6(3): e1551.

19. Kalantari E, Bandehpour M, Pazoki R, TaghipoorLailabadi N, Khazan H, Mosaffa N, Nazaripouya MR, Kazemi B. Application of recombinant Echinococcus granulosus antigen B to ELISA kits for diagnosing hydatidosis. Parasitology research 2010; 106(4): 847- 
851.

20. Rahimi H, Sadjjadi S, Sarkari B. Performance of antigen $\mathrm{B}$ isolated from different hosts and cyst locations in diagnosis of cystic echinococcosis. Iranian journal of parasitology 2011; 6(1): 12-19.

21. Sarkari B, Sadjjadi S, Abidi H, Izadpanah A, Kazemian S, Rafati A. Application of western blotting using native antigen $\mathrm{B}$ for serodiagnosis of human cystic echinococcosis. Iranian journal of parasitology 2007; 2(3): 7-12

22. Rott MB, Fernández V, Farias S, Ceni J, Ferreira HB, Haag KL, Zaha A. Comparative analysis of two different subunits of antigen B from Echinococcus granulosus: gene sequences, expression in Escherichia coli and serological evaluation. Acta tropica. 2000; 75(3):331-340.

23. Hernández-González A, Sánchez-Ovejero C, ManzanoRomán R, González Sánchez M, Delgado JM, PardoGarcía T, Soriano-Gálvez F, Akhan O, Cretu CM, Vutova K, Tamarozzi F, Mariconti M, Brunetti E, Vola A, Fabiani M, Casulli A, Siles-Lucas M. Evaluation of the recombinant antigens $\mathrm{B} 2 \mathrm{t}$ and $2 \mathrm{~B} 2 \mathrm{t}$, compared with hydatid fluid, in IgG-ELISA and immunostrips for the diagnosis and follow up of CE patients. PLoS neglected tropical diseases 2018; 12(9): e0006741.

24. Manzano-Román R, Sánchez-Ovejero C, HernándezGonzález A, Casulli A, Siles-Lucas M. Serological diagnosis and follow-up of human cystic echinococcosis: a new hope for the future? BioMed research international 2015; Article ID: 428205.

25. Jia H, Fan G, Yan Q, Liu Y, Yan Y, Jiang Z. High-level expression of a hyperthermostable Thermotoga maritima xylanase in pichia pastoris by codonoptimization. Journal of molecular catalysis B: enzymatic 2012; 78:72-77.

26. Burgess-Brown NA, Sharma S, Sobott F, Loenarz C,
Oppermann U, Gileadi O. Codon optimization can improve expression of human genes in Escherichia coli: A multi-gene study. Protein expression and purification 2008; 59(1): 94-102.

27. Hajizadeh MR, Mokarram P, Kamali Sarvestani E, Bolhassani A, Mostafavi Pour Z. Recombinant nonstructural 3 protein, rNS3, of hepatitis $\mathrm{C}$ virus along with recombinant GP96 induce IL-12, TNF $\alpha$ and a5integrin expression in antigen presenting cells. Hepatitis Mononthly 2013; 13(6): e8104.

28. Savardashtaki A, Sharifi Z, Hamzehlou S, Farajollahi MM. Analysis of immumoreactivity of heterologously expressed non-structural protein 4B (NS4B) from hepatitis $\mathrm{C}$ virus $(\mathrm{HCV})$ genotype 1a. Iranian journal of biotechnology 2016; 13(4): 32-37.

29. Gorjipour F, Sharifi Z, Samadikuchaksaraei A, Farajollahi MM, Hosseini A. Cloning, soluble expression and immunoreactivity of HIV-1 CRF35_AD p24 protein infusion with HP-thioredoxin from Iranian clinical isolates. Laboratory medicine 2012; 43(6): 245249.

30. Jiang L, Zhang YG, Liu MX, Feng Z. Analysis on the reactivity of five subunits of antigen $B$ family in serodiagnosis of echinococcosis. Experimental parasitology 2012; 131(1): 85-91.

31. Ahn CS, Cai H, Kim JG, Han X, Ma X, Bae YA, Yang HJ, Kang I, Wang H, Kong Y. An Echinococcus multilocularis antigen B3 proteoform that shows specific antibody responses to active-stage alveolar echinococcosis. Journal of clinical microbiology 2015; 53(10): 3310-3317.

32. Chen X, Zhang J, Feng X, Chen X, Yin S, Wen H, Zheng S. Humoural immune response and pathological analysis in patients with false immune diagnosis of cystic echinococcosis. Parasite immunology 2014; 36(4): 170-176. 